\title{
Carga objetiva y subjetiva en personas con enfermedad mental: evidencias empíricas
}

\author{
Maribel Cruz-Ortiz ${ }^{\star}$, Ma. del Carmen Pérez-Rodríguez ${ }^{\star \star}$, Elida Lisbeth Villegas-Moreno ${ }^{\star * \star}$, Noelia \\ Flores-Robaina $^{\star \star * \star}$, Eduardo Hernández-Ibarra ${ }^{\star \star \star \star *}$, Pedro Reyes-Laris ${ }^{\star * \star \star \star \star}$
}

* Doctora en Discapacidad. Profesora investigadora de tiempo completo, Facultad de Enfermería, Universidad Autónoma de San Luis Potosí, México.

Correo electrónico: redazul@hotmail.com

* Doctora en Ciencias de Enfermería. Coordinadora de la Maestría en Salud Pública. Profesora investigadora de tiempo completo, Facultad de Enfermería, Universidad Autónoma de San Luis Potosí, México.

Correo electrónico: salina67@hotmail.com

*** Estudiante de la Maestría en Salud Pública, Facultad de Enfermería, Universidad Autónoma de San Luis Potosí, México.

Correo electrónico: psic.elida@gmail.com

**** Doctora en Psicología, Facultad de Psicología, Universidad de Salamanca, España. Correo electrónico: nrobaina@usal.es

***** Doctor en Psicología. Profesor investigador de tiempo completo, Facultad de Enfermería, Universidad Autónoma de San Luis Potosí, México. Correo electrónico: eduardo.ibarra@uaslp.mx

****** Pasante de Licenciatura en Enfermería, Facultad de Enfermería, Universidad Autónoma de San Luis Potosí, México.

Correo electrónico: an_gg17@hotmail.com

\begin{abstract}
Resumen
Diversos estudios mencionan las consecuencias que genera el cuidado, especialmente si este se relaciona con la enfermedad mental. Este trabajo tuvo por objetivo analizar estudios publicados entre el 2003 y el 2010, relacionados con la carga percibida en cuidadores de pacientes con enfermedad mental. Se realizó una revisión sistemática de la literatura en bases de datos de acceso libre en Internet (Scielo, Pubmed y Lilacs). Los resultados indican que a menor edad del paciente, la carga hacia los cuidadores es mayor, independientemente del sexo o de su estado civil, utilizando como estrategia de afrontamiento la evitación y el apoyo social. Conclusiones: la carga varía a lo largo del tiempo y esta se ve influida por el tipo de sintomatología, siendo poco probable que mejore sin algún tipo de intervención.
\end{abstract}

Palabras clave: apoyo social, carga objetiva, carga subjetiva, cuidado, enfermedad mental.

\section{Objective and Subjective Burden in People with Mental Illness: Empirical Evidence}

\section{Abstract}

Diverse studies mention the consequences generated by providing special care to patients, particularly when associated with mental illness. The objective of this research was to analyze scientific studies published between 2003 and 2010 on the burden suffered by caregivers of patients with mental illnesses. A systematic review was made of literature found in open access databases (Scielo, Pubmed, Lilacs). The results suggest that, regardless of gender and marital status, when using strategies such as coping mechanisms and social support, younger patients have a greater impact on the burden felt by their caregivers than their older counterparts. This burden varies over time and is influenced by the type of symptomatology experienced by the patient and it is improbable that there will be improvement without some type of intervention.

Keywords: social support, objective burden, subjective burden, care, mental illness.

\section{Carga objetiva e subjetiva em pessoas com doença mental: evidências empíricas}

\section{Resumo}

Diversos estudos mencionam as consequências que gera o cuidado, especialmente se este se relaciona com a doença mental. Este trabalho teve por objetivo analisar estudos publicados entre 2003 e 2010, relacionados com a carga percebida em cuidadores de pacientes com doença mental. Realizou-se uma revisão sistemática da literatura em bases de dados de acesso livre na internet (Scielo, Pubmed e Lilacs). Os resultados indicam que a carga para os cuidadores é maior quando a idade do paciente for menor, independentemente do sexo ou de seu estado civil, utilizando como estratégia de enfrentamento a evitação e o apoio social. Conclui-se que a carga varia ao longo do tempo e esta se vê influenciada pelo tipo de sintomatologia, sendo pouco provável que melhore sem algum tipo de intervenção.

Palavras-chave: apoio social, carga objetiva, carga subjetiva, cuidado, doença mental. 
Estudios recientes como El cuidado de las personas: Un reto para el siglo XXI, muestran algunas de las consecuencias que genera el cuidado. En ellos es posible observar que los efectos positivos y negativos son en general semejantes pero varían de acuerdo con la etapa de la vida en la que se encuentra la persona cuidada (Tobío, 2008).

Otros estudios como Dependencia y solidaridad en las redes familiares, realizado en España, permiten ver que la afectividad es la razón por la que se cuida, predominantemente. Por otra parte, la participación de la familia en el cuidado disminuye cuando se compara el cuidado de mayores frente al cuidado de enfermos y personas con discapacidad, implicando en estos casos en mayor medida al Estado como corresponsable de dichos cuidados (Fernández y Tobío, 2007).

Investigaciones cualitativas marcan discursos sobre el cuidado, las cuales reflejan ambivalencia y pueden ordenarse gradualmente: desde el rechazo a cuidar (en el polo más negativo), pasando por la obligación y resignación (como compromiso ineludible y como deber) y, en el otro extremo, la satisfacción (sentimiento de utilidad social, autoestima o por afectividad-cariño) y lo vocacional. Este último es el más positivo pero infrecuente (Cruz, Pérez, Jenaro, Vega y Flores, 2010; Tobío, Agulló, Gómez y Martín, 2010).

Otros estudios muestran las repercusiones negativas que genera el cuidado, y que varían desde quienes afirman sentirse afectados por la ayuda económica prestada, el tiempo invertido y restado a la realización de su vida social o el disfrute del ocio, hasta quienes sienten sus efectos en la salud y el logro profesional (IEA, 2007).

\section{Método}

Se realizó una revisión sistemática de los artículos publicados sobre el tema de la carga percibida por cuidadores de personas con enfermedad mental, entre el 2003 y el 2010, en las bases de datos de acceso libre en Internet (Scielo, Pubmed y Lilacs), bajo los descriptores de cuidador, carga objetiva, carga subjetiva y enfermedad mental.

La selección de los artículos se limitó a aquellos que tuvieran como tema central al cuidador, que estuvieran disponibles, completos, y en idiomas español, portugués o inglés. Esta selección se hizo mediante una lectura rápida del resumen para identificar los datos antes mencionados; de los artículos que cumplieron los criterios se hizo la lectura íntegra para proceder a su análisis.
Para hacer el análisis se elaboró una matriz construida para este propósito identificando en ella el título, la fecha, el proceso metodológico y los resultados principales. Con esta información, los estudios fueron clasificados según correspondieran a: los síntomas del paciente y la carga en el cuidador; los factores predictores de carga; las características comunes de los cuidadores y la carga generada por el cuidado; instrumentos y técnicas de evaluación de carga, o intervenciones y apoyos proporcionados al cuidador primario.

\section{Resultados y discusión}

\section{Cargas objetiva y subjetiva}

Subyacen en estas percepciones y sensaciones lo que Hoenig y Hamilton (1966) diferenciaron como carga objetiva y subjetiva. Esta distinción es ampliamente utilizada en la investigación a pesar de existir múltiples definiciones de carga. La carga objetiva cubre todas aquellas cosas que el cuidador y su familia hacen (ayudar, supervisar, controlar, pagar, etcétera), experimentan (alteraciones en la rutina y relaciones interpersonales en la familia) y las restricciones en las actividades a las que están sometidos (ir de vacaciones, al club, practicar deportes, ir al trabajo, etcétera) como consecuencia de las tareas de cuidado. Por su parte, la carga subjetiva puede definirse como las actitudes y reacciones emocionales ante la experiencia de cuidar (Sales, 2003).

Algunos autores indican que las principales dimensiones de la carga objetiva son: efecto sobre el uso del tiempo libre, alteración de la rutina familiar, limitación de las actividades sociales y efecto sobre las relaciones dentro de la familia. Al respecto, la tabla 1, basada en un estudio de revisión sobre investigaciones aparecidas en el 2003 sobre personas con enfermedad mental severa, muestra los cambios más frecuentemente identificados tanto en la dimensión objetiva como subjetiva (Ohaeri, 2003). Este estudio coincide con otros que señalan que la severidad de los síntomas constituye un importante predictor de la carga (Awad y Voruganti, 2008; Lowyck, De Hert, Peeters, Wampers, Gilis y Peuskens, 2004; Papastavrou, Charalambous, Tsangari y Karayiannis, 2010).

\section{Enfermedad mental: los síntomas del paciente y la carga en el cuidador}

Entre las principales conductas asociadas con estos síntomas se indican mayoritariamente las asociadas con 
Tabla 1. Cambios objetivos y subjetivos en cuidadores de personas con enfermedad mental severa

\begin{tabular}{|l|l|}
\hline $\begin{array}{l}\text { Síntomas del paciente que } \\
\text { generan preocupación en los } \\
\text { cuidadores }\end{array}$ & $\begin{array}{l}\text { Disturbios nocturnos, agresividad, delirios, alucinaciones, abandono del cuidado personal, falta de coo- } \\
\text { peración, posible peligro para sí mismo u otros, los vecinos se quejan del comportamiento del paciente, } \\
\text { síntomas negativos como discapacidad y falta de productividad. }\end{array}$ \\
\hline Acciones de cuidado en casa & Control de síntomas; ayuda en la administración de medicamentos; mantenerlo activo todo el tiempo. \\
\hline $\begin{array}{l}\text { Interacciones con profesionales } \\
\text { de la salud }\end{array}$ & $\begin{array}{l}\text { Actuar como gestores en la asistencia buscando ayuda sin preocuparse por la atención profesional para } \\
\text { los problemas propios }\end{array}$ \\
\hline Acciones comunitarias & Obtención de recursos comunitarios adecuados. \\
\hline $\begin{array}{l}\text { Actitudes que causan angustia } \\
\text { emocional }\end{array}$ & $\begin{array}{l}\text { El estigma por asociación, dolor, culpa, impotencia, ira; necesidad de atención, dificultad para atribuir } \\
\text { significado a las acciones del paciente, sensación de pérdida. }\end{array}$ \\
\hline $\begin{array}{l}\text { Experiencias emocionalmente } \\
\text { edificantes }\end{array}$ & $\begin{array}{l}\text { Sensación de plenitud de derechos, auto-conocimiento a partir de la experiencia de cuidado, la familia } \\
\text { es fundamental para la esperanza; afrontamiento; satisfacción con los cuidados a pesar de la carga } \\
\text { objetiva. }\end{array}$ \\
\hline $\begin{array}{l}\text { Efectos sobre el funcionamien- } \\
\text { to familiar }\end{array}$ & Significativamente peor funcionamiento de la familia. \\
\hline Métodos de afrontamiento & $\begin{array}{l}\text { Empatía y la religiosidad fueron las estrategias más utilizadas, la forma de afrontamiento está influen- } \\
\text { ciada por las percepciones. }\end{array}$ \\
\hline $\begin{array}{l}\text { Morbilidad psiquiátrica en los } \\
\text { cuidadores }\end{array}$ & 25\% de los cuidadores tuvieron criterios de caso en el General Health Questionnaire. \\
\hline Modelos causales & Citada entre el 9 y 17\% de todas las culturas. El estrés psicosocial es el más comúnmente citado. \\
\hline $\begin{array}{l}\text { Tipos de asistencia profesional } \\
\text { requerido por la familia }\end{array}$ & $\begin{array}{l}\text { Mejorar el conocimiento médico de las enfermedades, tratamiento de conductas problemáticas, apoyo } \\
\text { social. }\end{array}$ \\
\hline
\end{tabular}

Fuente: elaboración propia a partir de Ohaeri, 2003

problemas de seguridad y posibles actos de violencia del paciente hacía sí mismo u otros, demandas excesivas, alta dependencia hacia el cuidador, alteraciones en los hábitos de sueño y conductas embarazosas. Probablemente porque las conductas que se presentan en las enfermedades mentales son compartidas por varios diagnósticos y constituyen la expresión de los síntomas, los niveles de carga y las formas de afrontamiento son semejantes en cuidadores de personas clínicamente estables con esquizofrenia y trastornos afectivos bipolares (Chadda, Singh y Ganguly, 2007).

En esta línea, un estudio realizado en Suiza, que analizó la relación entre carga y cuadros agudos de personas con esquizofrenia, encontró correlación entre amenazas, molestias, tiempo invertido con los afectados y carga relacionada con la restricción de la vida social y el ocio, pero no directamente con la agresión o el abuso de sustancias (Lauber, Eichenberger, Luginbühl, Keller y Rössler, 2003). Esto contrasta con los hallazgos encontrados en un estudio español según el cual el consumo de drogas se encuentra asociado con una mayor carga familiar, lo que pudiera obedecer a cuestiones de orden cultural (Ochoa et al., 2008).

Otras evidencias indican que la respuesta ante los síntomas del paciente parece estar diferenciada, encontrándose que los síntomas positivos del paciente se relacionan con la carga objetiva y con acciones de supervisión, en tanto que los síntomas negativos se asocian con la carga subjetiva que suscitan en el cuidador preocupación ante la situación de inacción del paciente y que generan comparativamente mayor carga (Ochoa et al., 2008; Provencher y Mueser, 1997). En ello coinciden Grandón, Jenaro y Lemos (2008), quienes señalan que la supervisión de conductas problema, aunque genera una menor carga objetiva que las actividades cotidianas, produce una mayor carga subjetiva. Esto indica que las conductas problema, a pesar de ser más ocasionales, preocupan en mayor medida a los cuidadores, sobre todo aquellos comportamientos relacionados con hetero y autoagresiones (Grandón et al., 2008).

Si bien la sintomatología psiquiátrica constituye un predictor de la carga, esta se ve incrementada cuando tales síntomas se presentan en personas que presentan comorbilidad. Así lo indican estudios realizados por ejemplo con personas con discapacidad intelectual en los que la presencia de síntomas psiquiátricos constituye una carga adicional para los cuidadores y condiciona la solicitud de ayuda externa (Maes, Broekman, Dosen y Nauts, 2003).

Uno de los autores que más ha contribuido a la definición operativa del concepto de carga es Schene (1990). Este autor, tras revisar las investigaciones existentes, distingue ocho áreas de la carga; las seis primeras están relacionadas con la carga objetiva, en tanto que las dos últimas, con la subjetiva.

La primera dimensión se refiere a las rutinas dentro de la casa, esto es, a los acuerdos recíprocos en el reparto de tareas que realiza la familia en sus relacio- 
nes cotidianas. Cuando un miembro no puede llevar a cabo estas tareas, otros deben asumir las funciones. El incremento del cuidado, la supervisión y el control del paciente por parte de la familia rompen la reciprocidad establecida. Los resultados de algunos estudios sugieren que las personas que tienen más necesidades de apoyo en la preparación de alimentos, el cuidado del hogar y las actividades durante el día de cuidado, causan una mayor carga familiar ya que los cuidadores tienen que supervisar las actividades de sus familiares enfermos en la vida cotidiana (Grandón et al., 2008; Ochoa et al., 2008).

La segunda área alude a las relaciones interpersonales entre los miembros de la familia y el paciente, en las que pueden aparecer tensiones. Estas se deben tanto a la necesidad de asumir el cuidado, como al estrés subjetivo experimentado por los miembros de la familia. En este sentido, estudios que han encontrado relación entre la calidad de vida y el deterioro en el área familiar, plantean la necesidad de incorporar intervenciones enfocadas hacia la reducción del estrés y la carga familiar, el aprendizaje de habilidades para la solución de problemas y de modos de afrontamiento eficaces que permitan el manejo de determinadas situaciones conflictivas que se dan en la convivencia diaria (Navarro, García-Heras, Carrasco y Casas, 2008).

En tercer lugar se encuentran las relaciones sociales, que pueden volverse tensas no sólo con la familia cercana sino también con los parientes, amigos, vecinos y conocidos. El efecto negativo sobre las relaciones se debe tanto al escaso tiempo disponible, como al estigma social que pesa sobre la enfermedad (Rose, Thornicroft, Pinfold y Kassam, 2007). La estigmatización puede segregar al paciente y generalizarse a toda la familia, de forma tal que se produzca una ruptura en las redes de apoyo social. A su vez, esta situación puede aumentar la vulnerabilidad del familiar a padecer un trastorno mental menor (por ejemplo, depresión).

A este respecto, un estudio que realizó una comparación entre la sobrecarga familiar y las redes sociales en personas con esquizofrenia y personas con enfermedades físicas crónicas, encontró que la carga objetiva fue semejante; sin embargo, la red social presente en las personas con enfermedad mental fue significativamente más débil. Los autores señalan como probables factores asociados: 1) mayor dificultad de los familiares para comprender el significado de los síntomas psiquiátricos, que los orgánicos; 2) los familiares tienden a sobrestimar la posibilidad de que el paciente controle los síntomas, lo que puede generar a su vez sentimientos de frustración y angustia; 3) el estigma asociado con la esquizofrenia sigue presente en general, especialmente respecto al riesgo de conductas agresivas; 4) escasa información correcta sobre las enfermedades mentales en los medios de comunicación (Magliano, Fiorillo, De Rosa y Maj, 2006).

La cuarta dimensión, tiempo libre y cuidados, plantea que asumir una mayor cantidad de tareas y ofrecer apoyo no sólo produce un deterioro en las relaciones sociales, sino también una disminución de las oportunidades del cuidador para el desarrollo de carrera y la realización de actividades recreativas como deportes, salir de vacaciones, participar en grupos, etcétera. En este sentido, se ha identificado una relación significativa entre redes sociales de apoyo familiar y profesional y carga, lo cual sugiere que la participación de los pacientes en programas diarios de respiro, etcétera, puede permitirle a los cuidadores preservar sus actividades independientes y evitar el aislamiento social (Jenaro, Flores y Caballo, 2008).

Estudios con población latinoamericana ponen de manifiesto la importante restricción del tiempo libre y vida privada en los familiares, así como los efectos negativos en la familia y las preocupaciones sobre el futuro del paciente (Grandón et al., 2008). Estos estudios identifican a la madre como la persona que muestra la mayor carga, aun si comparte la tarea con otros familiares, ya que es ella quien asume la mayoría del cuidado (Caqueo-Urízar y Gutiérrez-Maldonado, 2006; Jenaro et al., 2008).

En quinto lugar, las finanzas; se refieren a que la posición económica de la familia puede deteriorarse porque el paciente trabaja pocas horas o cesa de trabajar. Además, las necesidades de apoyo del miembro enfermo pueden obligar a que su cuidador deje de trabajar. A ello se debe de sumar la reducción en los ingresos derivada de los gastos en cuidados profesionales necesarios y de la conducta disruptiva del paciente en la economía familiar.

Estudios realizados en países en desarrollo muestran que la carga financiera generada tanto por los gastos médicos en la atención de la enfermedad como por la común falta de aporte económico en las personas con enfermedad mental severa genera una mayor carga en las personas de estratos sociales más bajos. Se señala además que muchas madres no trabajan debido a que deben permanecer en casa realizando el cuidado, con lo que el ingreso es aún más restringido. Esta situación parece repetirse incluso en cuidadores de países con mejores niveles de vida como España, donde las perso- 
nas con ingresos bajos, paradas e inactivas, tienen más probabilidades de dedicarse de forma intensiva a la tarea de dispensar cuidados de salud (Caqueo-Urízar y Gutiérrez-Maldonado, 2006).

Factores contribuyentes a las dificultades financieras se presentan en países cuyos sistemas sociosanitarios están escasamente extendidos o no brindan la protección necesaria a estas personas. En esta línea, un estudio realizado en Chile puso de manifiesto que sólo el $41 \%$ de la población de su estudio contaba con seguridad social (Caqueo-Urízar y Gutiérrez-Maldonado, 2006; Lowyck et al., 2004).

La sexta área, hijos y hermanos, plantea que cuando el paciente también es padre, puede ser necesario que otros miembros de la familia ayuden en su cuidado. Esta situación ha sido poco explorada en el tema que nos ocupa, dado el predominante perfil masculino de la enfermedad mental grave, particularmente de la esquizofrenia. Por ejemplo, un estudio cualitativo de Díaz-Caneja y Johnson (2004) con entrevistas en profundidad, realizado en Londres con mujeres con enfermedad mental severa que tenían al menos un hijo, mostró cómo las necesidades de apoyo aumentaban con la maternidad y crianza de los hijos.

Si bien en el estudio señalado arriba el Estado proporcionaba un importante apoyo, en otro contexto probablemente se desplazaría a la familia, pues, como se indica en el mencionado estudio: "Es muy difícil, cuando usted está envuelto en sus propias necesidades emocionales, ver las necesidades emocionales de sus hijos". Otras mujeres señalaron que sus habilidades de crianza se vieron dañadas en gran medida durante las recaídas de la enfermedad. Los efectos de la enfermedad y la conducta asociada en los hijos es identificada claramente por estos pacientes; así, una de las entrevistadas señala: "Cuando no puedo levantarme, o me siento muy mal, he tirado el medicamento, mi hijo tiene que estar a cargo, él tiene que saber lo que hay que hacer" (Díaz-Caneja y Johnson, 2004).

Cuando el paciente es el hijo, sus hermanos suelen recibir menor atención por parte de los padres que están ocupados del cuidado del enfermo. Además, pueden sentir la presión de tener que ser más serviciales, asumir funciones extras o brindar más apoyo. Los problemas familiares, el estigma social y el aislamiento pueden tener los mismos efectos en el bienestar físico y psicológico de los hermanos que en otros miembros de la familia. Algunos estudios constatan que la carga generada por la asistencia cotidiana en actividades de la vida diaria es mayor para progenitores y herma- nos de personas con enfermedad mental desocupadas, solteras o con síntomas residuales, lo cual es más frecuente en personas con largo tiempo de evolución con la enfermedad.

En séptimo lugar, la salud alude a que cuidar a una persona con enfermedad mental puede tener consecuencias para la salud física y mental de los miembros de la familia involucrados. Estas consecuencias son normalmente el resultado del estrés crónico derivado del cuidado. En este sentido es muy difícil discernir claramente aquellos síntomas pertenecientes a trastornos psicosomáticos, diferenciándolos de las enfermedades "físicas" y llegar a conclusiones sobre su posible origen en la carga que representa el cuidado del enfermo (Jenaro et al., 2008).

La última y octava dimensión, el estrés subjetivo, incluye un amplio rango de sentimientos y emociones negativas como la ambivalencia, la rabia, la compasión, la preocupación por el futuro y los sentimientos de pérdida. Sentimientos como estar enojado cuando se encuentra cerca del paciente, experimentar tensión y preocupación por ambos y, en general, sensación de pérdida de control de la propia vida desde la aparición de la enfermedad del paciente, son comunes en los familiares (Caqueo-Urízar y Gutiérrez-Maldonado, 2006).

\section{Los factores predictores de carga}

Las áreas señaladas y en las que predominantemente se identifica la carga (Schene, 1990) se encuentran relacionadas en general con el cuidador, con la persona cuidada y con su contexto, pero se componen de múltiples factores que de manera específica se han estudiado, y un grupo de ellos han sido identificados como predictores de carga (figura 1).

Pese a que estos predictores no gozan de un consenso absoluto dadas las limitaciones metodológicas de los estudios predominantemente transversales y con dificultades para emparejar los grupos de pacientes y familiares, la evidencia empírica los señala como posibles puertas de entrada para la puesta en marcha de estrategias de abordaje que palíen la carga sobre todo cuando se aplican de forma temprana (Boatas, Solivellas, Méndez, Burillo y Cubero, 2007; Grandón et al., 2008; Möller-Leimkühler y Obermeier, 2008; Rebolleda y Soladana, 2008).

Es necesario, no obstante, desarrollar un mayor número de estudios para demostrar fehacientemente la influencia en la carga de las intervenciones dirigidas 


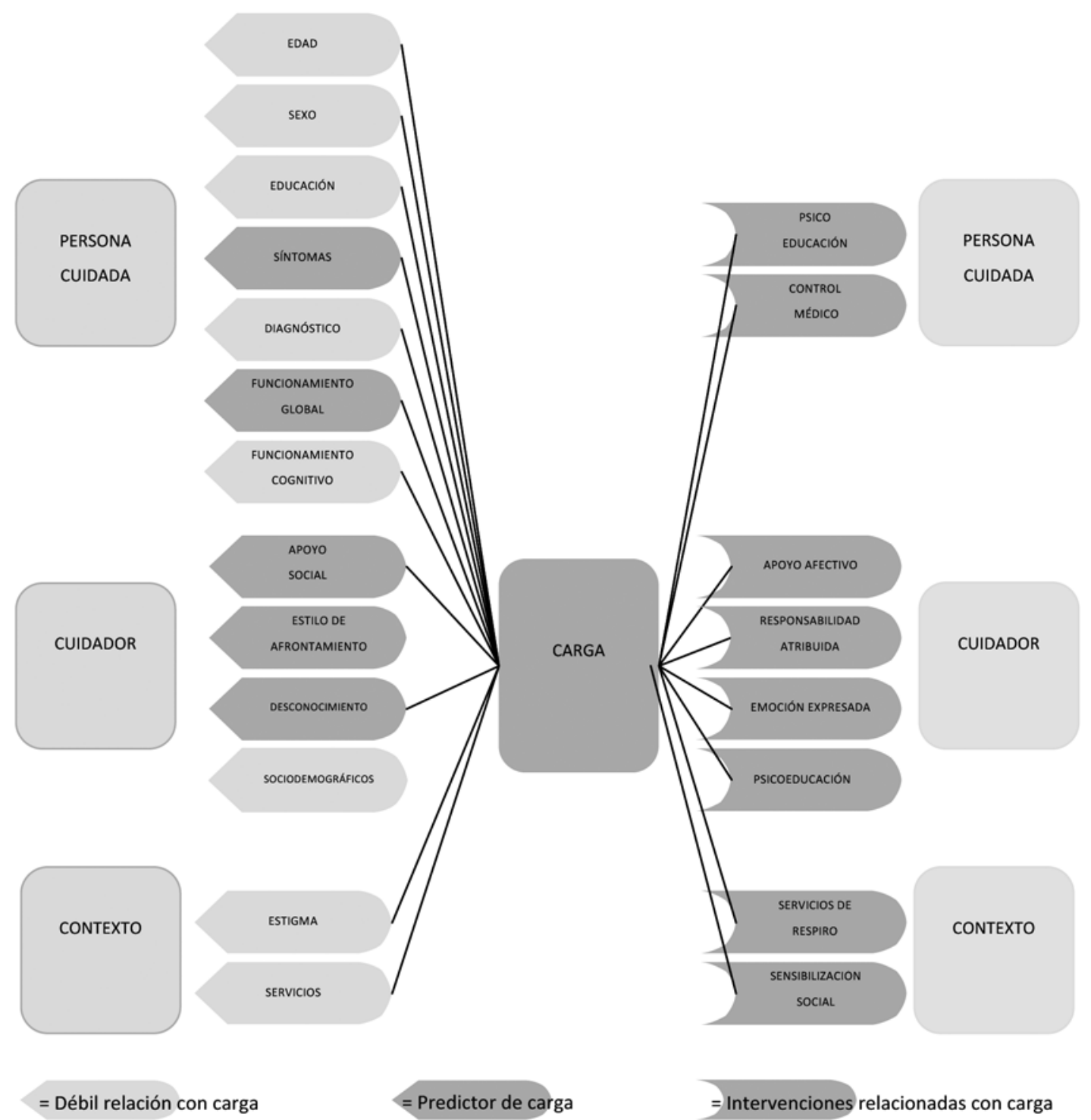

Figura 1. Factores predictores de carga e intervenciones Fuente: elaboración propia

al contexto y a la propia persona con enfermedad mental (Moriana, Alarcón y Herruzo, 2004).

Datos como los que aportan Möller-Leimkühler y Obermeier (2008) en su estudio longitudinal, permiten afirmar que las características del paciente parecen tener una menor contribución a la carga que las características familiares y del cuidador (Hadrys, Adamowski y Kiejna, 2010; Hjärthag, Helldin, Karilampi y Norlander, 2010; Möller-Leimkühler, 2005; MöllerLeimkühler y Obermeier, 2008), y depende en particular del grado de control percibido, la respuesta negativa al estrés y los factores estresantes de la vida (Chadda et al., 2007).

\section{Características comunes de los cuidadores y la carga generada por el cuidado}

Una revisión de estudios publicados en los últimos cinco años nos permite elaborar un perfil aproximado de las personas con enfermedad mental severa. Así, en su mayoría son hombres, con diagnóstico de esquizofrenia, solteros, que se encuentran viviendo con la familia, generalmente con los padres, con la madre como principal proveedora de cuidado, sin empleo (Joffre-Velázquez, García-Maldonado, Saldívar-González, Martínez-Perales y Medina-Medina, 2009; López, 2007). 
Además de este perfil del paciente, la tabla 2 resume las características comunes de los cuidadores y la carga generada por el cuidado.

Si bien no se trata de un análisis exhaustivo, y los estudios seleccionados difieren respecto a los instrumentos utilizados, las características de la muestra y el contexto cultural o social que modifica el entendimiento de la enfermedad o la disposición de servicios, son precisamente estas diferencias las que nos dan una pauta para encontrar nuevas líneas de investigación a nivel local.

Tabla 2. Hallazgos en estudios relacionados con sobrecarga y cuidadores de personas con enfermedad mental grave

Estudio: Burden of care in families of patients with schizophrenia, realizado en Chile (Caqueo-Urízar y Gutiérrez-Maldonado, 2006).

Hallazgos con datos sociodemográficos. La carga no se asoció con sexo o estado civil del cuidador. Tampoco con el sexo, nivel educativo o empleo de los pacientes, ni con el tiempo viviendo juntos o el número total de episodios de la enfermedad.

Hallazgos sobre el cuidador.1) a menor edad del paciente, se encontró mayor carga del cuidador; 2) cuidadores con mayor nivel educativo tienen menos carga; 3) evaluados con escala Zarit nivel de carga intensa en 90,2\%; 4) la carga se incrementa conforme aumenta la edad del cuidador; 5) la carga es mayor en cuidadores sin trabajo fuera del hogar.

Servicios. Escasez de servicios residenciales; el $41 \%$ recibe apoyo gubernamental sanitario.

Estudio: A prospective study of relationship between burden and coping in caregivers of patients with schizophrenia and bipolar affective disorder, realizado en la India (Chadda et al., 2007).

Hallazgos con datos sociodemográficos. No hubo diferencias significativas en las puntuaciones de carga total relacionadas con edad, sexo, educación, tipo familiar, diagnóstico, ingresos, ocupación o residencia. No se identificaron variaciones en la carga en los seis meses.

Hallazgos sobre el cuidador. 1) evaluados con Burden Assessment Schedule, los cuidadores utilizaron como estrategias de afrontamiento más comunes la evitación y el apoyo social; 2) se encontró correlación entre carga total y factores de carga.

Servicios. Se señala una red de servicios sanitarios y sociales escasamente estructurada.

Estudio: Burden on caregivers of people with schizophrenia: comparison between Germany and Britain (Roick et al., 2007).

Hallazgos con datos sociodemográficos. Dos tercios de los pacientes viven con los padres. La carga familiar se asoció con síntomas del paciente, sexo masculino, desempleo, estado civil y tamaño de red social. La carga fue mayor en Gran Bretaña a pesar de tener menos contacto pacientecuidador.

Hallazgos sobre el cuidador. 1) evaluados con Involvement Evaluation Questionnaire; 2) la mayor carga se asoció con preocupaciones sobre el paciente y tener que animarlo a hacer cosas; 3) el factor de menos peso se relacionó con tareas de supervisión; 4) la carga se asoció con habilidades de afrontamiento y presencia de red social; 5) el mayor contacto con el paciente se asoció con mayor carga; 6) los cuidadores casados y a cargo de su cónyuge mostraron mayor carga.

Servicios. Se cuenta con una extensa protección sociosanitaria, aunque existen diferencias entre los dos países comparados, lo que se refleja en el indicador de satisfacción general con los servicios de salud, que es superior en Alemania.

Estudio: Do needs, symptoms or disability of outpatients with schizophrenia influence family burden? España (Ochoa et al., 2008).

Hallazgos con datos sociodemográficos. La discapacidad y ser hombre representaron mayores niveles de carga familiar. Las necesidades de los pacientes que más se asociaron con la carga familiar fueron las actividades de la vida diaria, drogas y alcohol, autocuidado, administración del dinero y cuidado de la casa.

Hallazgos sobre el cuidador. 1) evaluados con Camberwell Assessment of Needs y Objective and Subjective Family Burden Interview; 2) el número de necesidades de la vida diaria de los pacientes y niveles más altos de psicopatología se correlacionaron con niveles más altos de carga familiar; 3) la respuesta ante los síntomas positivos del paciente está relacionada con acciones de supervisión, en tanto que para los síntomas negativos se asocia con la preocupación.

Fuente: elaboración propia

\section{Instrumentos y técnicas de evaluación de carga}

Parte de las dificultades metodológicas para evaluar la eficacia de las intervenciones dirigidas a disminuir la carga radican en las múltiples conceptualizaciones que se tienen del fenómeno de la carga en los cuidadores. Así, estudios de revisión de instrumentos que miden carga del cuidador (Reine et al., 2004) han podido identificar hasta diez dimensiones distintas. Estas son:

- Percepción de la conducta del paciente por parte del cuidador.

- Relaciones con el paciente (esta área incluye aspectos cualitativos de la relación y el apoyo prestado por el cuidador, pero muy pocos instrumentos han 
incluido aspectos positivos generados por la relación de cuidado, señalándola como experiencia personal positiva).

- Vida cotidiana del cuidador (actividades domésticas, actividades profesionales, finanzas, entretenimiento).

- Vida familiar (relaciones matrimoniales, relaciones familiares y el efecto sobre los niños).

- Vida social (amistades, relaciones de vecindad, estigma).

- Salud física.

- Salud mental.

- Uso del sistema de cuidados (satisfacción con el sistema de atención y relaciones con el equipo de salud).

- Experiencia subjetiva.

- Anticipación (perspectivas, proyección para el futuro, filosofía de vida.

Algunos instrumentos incluyen una valoración global que puede centrarse en la experiencia general del cuidador y considerarla como una medida general de malestar. Esta medida es cuestionada en algunos estudios por sus dificultades para establecer límites tem- porales y distinguir con ello entre la carga presente y los cambios percibidos desde el inicio de la enfermedad del paciente. Otros instrumentos como el Experience of Caregiving Inventory (ECI) permiten obtener puntuaciones globales por medio de la suma de sus diferentes factores teniendo en cuenta las dimensiones de carga objetiva y subjetiva (Reine, et al., 2004).

Tales conceptualizaciones se reflejan en una amplia gama de instrumentos que han sido construidos con propósitos y poblaciones diferentes, y que si bien permiten visualizar varias facetas de la carga, han sido cuestionados por la variación en la robustez de sus propiedades psicométricas y la validez de sus resultados (Reine, Lancon, Simeoni, Duplan y Auquier, 2003).

Estudios que evalúan y comparan las características de diferentes instrumentos han propuesto introducir nuevos criterios de evaluación, y contrastaron la mayor utilidad de instrumentos generales frente a los centrados en patologías o condiciones particulares de la persona cuidada (Schulze y Rössler, 2005).

Un análisis presentado por Harvey et al. (2008) acerca de estos nuevos criterios propuestos y de algunos instrumentos que reúnen mejores propiedades avala la necesidad de difundirlos. Y ello porque al tratarse

Tabla 3. Objetivos y criterios para evaluar instrumentos de carga

\begin{tabular}{|c|c|c|c|c|}
\hline \multicolumn{2}{|c|}{ Objetivos frecuentes } & \multicolumn{3}{|c|}{ Criterio de evaluación } \\
\hline \multicolumn{2}{|c|}{$\begin{array}{l}\text { 1. Evaluar consecuencias de la carga } \\
\text { - Influencia en la salud del cuidador } \\
\text { - Impacto financiero } \\
\text { - Uso de servicios de salud } \\
\text { - Calidad de vida del cuidador }\end{array}$} & $\begin{array}{l}\text { A } \\
\text { B. } \\
\text { C }\end{array}$ & \multirow{2}{*}{\multicolumn{2}{|c|}{$\begin{array}{l}\text { Instrumento desarrollado para evaluar el constructor } \\
\text { cuidado } \\
\text { El desarrollo de ítems involucró cuidadores } \\
\text { Presenta evidencia sobre aceptación del instrumento por } \\
\text { cuidadores } \\
\text { Adecuado para usarse en cuidadores de personas con } \\
\text { enfermedad mental o demencia } \\
\text { Fiabilidad } \\
\text { Validez } \\
\text { Capacidad de respuesta } \\
\text { Precisión } \\
\text { Viabilidad de aplicación } \\
\text { Facilidad de interpretación }\end{array}$}} \\
\hline \multicolumn{2}{|c|}{$\begin{array}{l}\text { 2. Identificar predictores de carga } \\
\text { - Tipo de enfermedad } \\
\text { - Tipo de relación cuidador-paciente } \\
\text { - Influencia de los síntomas } \\
\text { - Déficit relacionados con la enfermedad } \\
\text { - Características sociodemográficas del paciente } \\
\text { - Redes de apoyo } \\
\text { - Características sociodemográficas del cuidador }\end{array}$} & $\begin{array}{l}\text { Adecuado para usarse en cuidadores de personas con } \\
\text { enfermedad mental o demencia } \\
\text { Fiabilidad } \\
\text { Validez } \\
\text { Capacidad de respuesta } \\
\text { Precisión } \\
\text { Viabilidad de aplicación } \\
\text { Facilidad de interpretación }\end{array}$ & & \\
\hline \multicolumn{2}{|l|}{$\begin{array}{ll}\cdot & \mathrm{I} \\
\bullet & \mathrm{I} \\
\bullet & \mathrm{F} \\
- & \mathrm{C}\end{array}$} & & & \\
\hline \multicolumn{5}{|c|}{ Instrumentos mejor puntuados (entre paréntesis se muestran los criterios cumplidos) } \\
\hline $\begin{array}{l}\text { - Screen for Caregiver Burden } \\
\text { - (Vitaliano, Maiuro, Ochs y } \\
\text { Russo) } \\
\text { - }(\text { A,B,D,E,F,G,I,J) } \\
\text { - Mide: Carga }\end{array}$ & $\begin{array}{l}\text { - Revised Scale for Caregiving } \\
\text { Self-Efficacy } \\
\text { - (Zeiss1, Gallagher-Thompson, } \\
\text { Lovett, Rose y McKibbin) } \\
\text { - (A,C,D,E,F,H,J) } \\
\text { - Mide: Confianza en el cuidado }\end{array}$ & $\begin{array}{l}\text { - } \text { Picot } \\
\text { - } \text { (Pico } \\
\text { - } \text { (A,B } \\
\text { - Mide } \\
\text { cuida }\end{array}$ & $\begin{array}{l}\text { aregiver Rewards Scale } \\
\text { Youngblut y Zeller) } \\
\text { S.F,G,H,I,J) } \\
\text { Sentimientos sobre el } \\
\text { o }\end{array}$ & $\begin{array}{l}\text { - Involvement Evaluation Ques- } \\
\text { tionnaire } \\
\text { - (Schene y Wijngaarden) } \\
\text { - (A,C,D,E,F,G,I) } \\
\text { - Mide: Múltiples constructos } \\
\text { (carga, cuidado, estrés) }\end{array}$ \\
\hline
\end{tabular}

Fuente: elaboración propia a partir de Harvey et al., 2008 
de los de más reciente creación, han sido utilizados en muy pocos estudios frente a instrumentos tradicionales como el Zarit, que sigue siendo el más utilizado en investigaciones de carga.

En la tabla 3 se ofrece un resumen sobre estos nuevos criterios de evaluación, entre los que se destacan la participación de cuidadores en su desarrollo y las posibilidades de aplicar el instrumento en la práctica cotidiana más allá de su utilidad para la investigación.

Estos criterios son de particular relevancia a la hora de elegir instrumentos que se adecuen tanto al objetivo y propósito del estudio como a la solidez psicométrica y población de referencia. Ello posibilita la generación de estudios en áreas que han recibido menos atención, como las relacionadas con el contexto y sus posibles ámbitos de intervención (Awad y Voruganti, 2008).

\section{Intervenciones y apoyos proporcionados al cuidador primario}

En consecuencia, las intervenciones y los apoyos proporcionados a la familia se convierten en un mediador entre el cuidado brindado y la carga generada por este cuidado. Ello explica que la carga se esté empleando como una variable de resultado en la evaluación de los servicios de salud. Dicho de otra forma, en la medida en que estos apoyos brindan a la persona con enfermedad mental mayores posibilidades de vida independiente, el cuidado es compartido entre diversas instancias y, en consecuencia, también la carga (Bover y Gastaldo, 2005; Magliano, Fiorillo, Malangone, Marasco, Guameri y Maj, 2003).

La familia requiere apoyos variados, encaminados a responder a diferentes necesidades. Así podemos enunciar los relacionados con el apoyo físico por medio de servicios de respiro o descanso, con el apoyo psicológico (emocional, atención, orientación), el apoyo social (formación e información, revalorización) y el económico (subvenciones). Dentro de esta variedad de intervenciones se abordarán las relacionadas con el apoyo psicológico, pues el resto han sido tratadas al referirnos a intervenciones relacionadas con la persona cuidada.

También cabe mencionar que dada la ya señalada importancia que tiene la presencia de la sintomatología y su relación con la carga, todas estas intervenciones se desarrollan en conjunto con el tratamiento médicopsiquiátrico (Kane, 2006) para facilitar el ajuste a la experiencia del cuidado y permitir a la familia llevar una vida lo más normal posible.
Las intervenciones más comunes incluyen variedades psicoeducativas a través de grupos de discusión, grupos de apoyo y asesoramiento. La psicoeducación es la denominación más común para aludir a un conjunto de intervenciones que combinan la prestación de información con objetivos terapéuticos, enfocándose en ofrecer información sobre la enfermedad, modificar ciertas respuestas emocionales, reducir el contacto paciente-familia, mejorar la comunicación, dotar a las familias de estrategias válidas de afrontamiento y resolución de problemas y en ofrecer apoyo (Fernández, 2010).

Concretamente, lograr que los familiares conozcan la enfermedad y entiendan su alcance y repercusión en la vida cotidiana, unido al aprendizaje en resolución de problemas y a cambios en su manera de comunicarse, puede contribuir a reducir el riesgo de recaídas del familiar afectado y favorecer su recuperación (Fernández, 2010). En esta línea, se estima que tal reducción de recaídas disminuiría hasta en un $20 \%$, reflejándose en los días de estancia y el número de reinternamientos si se incluyera a los familiares en los tratamientos (Bäuml, Froböse, Kraemer, Rentrop y Pitschel-Walz, 2006; Murray-Swank y Dixon, 2004; Rummel-Kluge y Kissling, 2008).

Estudios como el de Gutiérrez-Maldonado y Caqueo-Urízar (2007) con población latinoamericana muestran cómo estas intervenciones pueden contribuir a disminuir la carga familiar, especialmente en las madres y en los menores. Además, es probable que el efecto se vea incrementado por el mayor contacto que se genera durante la intervención entre cuidadores y profesionales (Magaña, Ramírez, Hernández y Cortez, 2007). También se ha evidenciado la mayor efectividad de la intervención en personas con menor nivel educativo, lo que es explicado con base en que dicho estrato es el que recibe con menor frecuencia información y orientación sobre el manejo de la enfermedad (Gutiérrez-Maldonado y Caqueo-Urízar, 2007).

Los programas psicoeducativos dirigidos a mejorar estrategias de afrontamiento de los cuidadores, consistentes en la reducción de conductas de evitación o resignación hacia el cuidado del paciente y en el aumento de actitudes positivas, se han mostrado efectivos en la disminución de la carga incluso después de varios años. Por otra parte, la participación del cuidador en grupos de autoayuda representa la integración en un ambiente en el que pueden compartir experiencias y estrategias de afrontamiento con otras familias (Chien y Norman, 2009; Díaz et al., 2005).

Otras intervenciones psicoeducativas con familias también han reportado beneficios para disminuir 
la carga. Por ejemplo, las intervenciones con la familia ante las recaídas de las personas con esquizofrenia desde el primer día del ingreso hospitalario y hasta su alta, ponen de manifiesto la reducción de estos sentimientos de carga en la familia (Berglund, Olof y Edman, 2003).

Aunque, como hemos visto, las características de cuidadores y personas cuidadas parecen ser semejantes, no es menos importante señalar que el contexto en el que se desarrollan modifica esta relación, y por tanto las intervenciones también deben ser matizadas por estas diferencias. Una muestra de la necesidad de realizar más estudios desde una diversidad de enfoques deriva del metaanálisis realizado por Eack y Newhill (2007) sobre la relación entre síntomas psiquiátricos y calidad de vida. En dicho estudio se confirma la existencia de una relación negativa entre psicopatología y calidad de vida, si bien dicho factor sólo explica el $12 \%$ de la varianza de calidad de vida.

Otros estudios sobre predictores de carga familiar (Möller-Leimkühler y Obermeier, 2008) ratifican la sintomatología como factor asociado con la carga, y también ponen de manifiesto que las características individuales de los cuidadores, como el estilo de afrontamiento y las respuestas ante el estrés, requieren intervenciones a largo plazo (Breitborde, López y Kopelowicz, 2010; Grandón et al., 2008; Roick, Heider, Toumi y Angermeyer, 2006; Vidal, Cortés, Valero, Gutiérrez-Zotes y Labad, 2008).

\section{Conclusiones}

Por medio de estas evidencias empíricas es posible afirmar que cuidar es una actividad compleja que requiere habilidades, entre otras, de empatía, paciencia, dedicación y esfuerzo, tanto físico como psicológico. En consecuencia, ello se traduce para quienes la desempeñan en cansancio y, a menudo, en patologías directamente relacionadas con su dedicación. Por otra parte, los hallazgos presentados apoyan las siguientes premisas señaladas por Magliano et al. (2006):

1. La carga puede variar a lo largo del tiempo.

2. La carga está influida por el tipo de sintomatología.

3. Es poco probable que sin intervenciones mejore la situación de la carga.

Respecto a la identificación de factores que contribuyen a la carga y requieren intervenciones, estudios que evalúan carga en cuidadores de personas con esquizofrenia, como el de Reine et al. (2004), han identificado cuatro áreas generales:

1. Sobre la información (respecto a la enfermedad, el tratamiento, la asistencia a las partes interesadas, los dispositivos de atención compatibles).

2. En torno a las relaciones con el equipo de atención médica (presente o ausente, calidez, comprensión, suficiencia).

3. Respecto a la confianza y generación de satisfacción o frustración por los servicios de apoyo (ocio, viviendas protegidas, optimización del tratamiento).

4. Relacionada con las dificultades enfrentadas durante las situaciones de crisis.

Como es posible observar, si bien estas áreas reflejan necesidades, percepciones y expectativas del cuidador, se centran en la relación con los profesionales que proveen servicios. Por esta razón, como se ha señalado, la carga es considerada cada vez más como parte de los indicadores de evaluación de servicios a pesar de que esta relación haya sido aún poco estudiada.

Por otra parte, es interesante señalar que la introducción de factores positivos en la relación de cuidado e incorporados en algunos instrumentos de medición de carga está llevando cada vez más a intentar evaluar la carga, incorporándola en el constructo de calidad de vida.

\section{Referencias}

Awad, A. y Voruganti, L. (2008). The burden of schizophrenia on caregivers: a review. Pharmacoeconomics, 26(2), 149-162.

Bäuml, J., Froböse, T., Kraemer, S., Rentrop, M. y PitschelWalz, G. (2006). Psychoeducation: a basic psychotherapeutic intervention for patients with schizophrenia and their families. Schizophr Bull, 32(supp 1), S1-S9. doi: $10.1093 / \mathrm{schbul} / \mathrm{sbl017}$

Berglund, N., Olof, J. y Edman, A. (2003). Family intervention in schizophrenia: Impact on family burden and attitude. Soc Psychiat Epidemiol, 38(3), 116-121.

Boatas, F., Solivellas, A., Méndez, I., Burillo, I. y Cubero, L. (2007). Programa PAPED: implantación de un programa de atención a primeros episodios psicóticos. Informaciones Psiquiátricas, 187, 19-36.

Bover, A. y Gastaldo, D. (2005). A centralidade da família como recurso no cuidado domiciliar: perspectivas de gênero e geração. Ver. Bras. Enferm., 58(1), 9-16. 
Breitborde, N., López, S. R. y Kopelowicz, A. (2010). Expressed emotion and health outcomes among MexicanAmericans with schizophrenia and their caregiving relatives. J Nerv. Ment. Dis., 198(2), 105-109.

Caqueo-Urízar, A. y Gutiérrez-Maldonado, J. (2006). Burden of care in families of patients with schizophrenia. Quality of Life Research, 15(4), 719-724.

Chadda, R., Singh, T. y Ganguly, K. (2007). Caregiver burden and coping: A prospective study of relationship between burden and coping in caregivers of patients with schizophrenia and bipolar affective disorder. Soc Psychiatry Psychiatr Epidemiol, 42(11), 923-930.

Chien, W. y Norman, I. (2009). The effectiveness and active ingredients of mutual support groups for family caregivers of people with psychotic disorders: a literature review. Int. J Nurs Stud, 46(12), 1604-1623.

Cruz, M., Pérez, M. C., Jenaro, C., Vega, V. y Flores N. (2010). Vivenciando las necesidades de apoyo en la enfermedad mental. Index de Enfermería, 20(1-2), 16-20.

Díaz-Caneja, A. y Johnson, S. (2004). The views and experiences of severely mentally ill mothers. A qualitative study. Social Psychiatry and Psychiatric Epidemiology, 39(6), 472-482.

Díaz, L., Ortega, H., Leaños, C., Rodríguez, M. S., Rascón, M. L., Valencia, M., Gutiérrez, M. L, Mares, M. E. y Sánchez, A. (2005). La rehabilitación integral del paciente esquizofrénico en México: El modelo del Instituto Nacional de Psiquiatría Ramón de la Fuente. Salud Mental, 28(6), 9-19.

Eack, S. y Newhill, C. (2007). Psychiatric Symptoms and Quality of Life in Schizophrenia: A Meta-Analysis. Schizophrenia Bulletin, 33(5), 1225-1237.

Fernández, J. (2010). Modelos de intervención con familias de personas afectadas por una enfermedad mental grave y duradera. Infocope online. Recuperado de http:// www.infocop.es/view_article.asp?id=2880\#inicio

Fernández, J. A. y Tobío, C. (2007). Andalucía: dependencia $y$ solidaridad en las redes familiares. Recuperado de http://www.juntadeandalucia.es:9002/redesfamiliares/ redesFamiliares.pdf

Grandón, P., Jenaro,C. y Lemos, S. (2008). Primary caregivers of schizophrenia outpatients: Burden and predictor variables. Psychiatry Research-Neuroimaging, 158(3), 335343.

Gutiérrez-Maldonado, J. y Caqueo-Urízar A. (2007). Effectiveness of a psycho-educational intervention for reducing burden in latin american families of patients with schizophrenia. Quality of Life Research, 16(5), 739-747.

Hadrys, T., Adamowski, T. y Kiejna, A. (2010). Mental disorder in Polish families: is diagnosis a predictor of caregiver's burden? Soc Psychiat Epidemiol, 46(5), 363-372.
Harvey, K., Catty, J., Langman, A., Winfield, H., Clement, S., Burns, E., White, S. y Burns, T. (2008). A review of instruments developed to measure outcomes for carers of people with mental health problems. Acta Psychiatrica Scandinavica, 117(3), 164-176.

Hjärthag, F., Helldin, L., Karilampi, U. y Norlander, T. (2010). Illness-related components for the family burden of relatives to patients with psychotic illness. Soc Psychiat Epidemiol, 45(2), 275-283.

Hoenig, J. y Hamilton, M. (1966). The schizophrenic patient in the community and his effect on the household. International Journal of Social Psychiatriy, 12(3), 165-176.

IEA. (2007). Andalucía: dependencia y solidaridad en las redes familiares. Sevilla: Instituto de Estadística de Andalucía.

Jenaro, C., Flores, N. y Caballo, C. (2008). Necesidades de cuidadores de personas en situación de dependencia en la provincia de Salamanca. Salamanca: Fundación Memoria de D. Samuel Solórzano Barruso.

Joffre-Velázquez, V., García-Maldonado, G., Saldívar-González, A., Martínez-Perales, G. y Medina-Medina O. (2009). Perfil de pacientes admitidos en un Hospital Psiquiátrico Mexicano para su tratamiento y rehabilitación en el año 2008. Resultados preliminares. Alcmeon, 15(4), 296-303.

Kane, J. (2006). Review of treatments that can ameliorate nonadherence in patients with schizophrenia. J Clin Psychiatry, 67(suppl 5), 9-14.

Lauber, C., Eichenberger, A., Luginbühl, P., Keller, C. y Rössler, W. (2003). Determinants of burden in caregivers of patients with exacerbating schizophrenia. European Psychiatry, 18(6), 285-289.

López, F. (2007). Calidad de vida en pacientes psicóticos externos. Alcmeon, 14(2), 65-75.

Lowyck, B., De Hert, M., Peeters, E., Wampers, M., Gilis, P. y Peuskens, J. (2004). A study of the family burden of 150 family members of schizophrenic patients. European Psychiatry, 19(7), 395-401.

Maes, B., Broekman, T., Dosen, A. y Nauts, J. (2003). Caregiving burden of families looking after persons with intellectual disability and behavioural or psychiatric problems. Journal of Intellectual Disability Research, 47(6), 447-455.

Magaña, S., Ramírez, J., Hernández, M. y Cortez, R. (2007). Psychological distress among latino family caregivers of adults with schizophrenia: The roles of burden and stigma. Psychiatric Services, 58(3), 378-384.

Magliano, L., Fiorillo, A., De Rosa, C. y Maj, M. (2006). Family burden and social network in schizophrenia vs.physical diseases: preliminary results from an Italian national study. Acta Psychiatr Scand, 113(supp 429), 60-63. 
Magliano, L., Fiorillo, A., Malangone, C., Marasco, C., Guameri, M. y Maj, M. (2003). The Effect of Social Network on Burden and Pessimism in Relatives of Patients With Schizophrenia. American Journal of Orthopsychiatry, 73(3), 302-309.

Möller-Leimkühler, A. (2005). Burden of relatives and predictors of burden. Baseline results from the Munich 5-year-follow-up study on relatives of first hospitalized patients with schizophrenia or depression. Eur Arch Psychiatry Clin Neurosci, 255(4), 223-231.

Möller-Leimkühler, A. y Obermeier, M. (2008). Predicting caregiver burden in first admission psychiatric patients: 2 years follow-up results. Eur Arch Psychiatric Clin Neurosci, 258(7), 406-413.

Moriana, E., Alarcón, E. y Herruzo, J. (2004). Tratamiento combinado de la esquizofrenia aplicado en el ámbito domiciliario. Psicothema, 16(3), 436-441.

Murray-Swank, A. y Dixon, L. (2004). Family psychoeducation as an evidence-based practice. CNS Spectr, 9(12), 905-912.

Navarro, D., García-Heras, S., Carrasco, O. y Casas, A. (2008). Calidad de vida, apoyo social y deterioro en una muestra de personas con trastorno mental grave. Intervención Psicosocial, 17(3), 321-336.

Ochoa, S., Vilaplana, M., Haro, J., Vilalta, V., Martínez, F., Negredo, M., Casacuberta, P., Paniego, E., Usall, J., Dolz, M. y Autonell, J. (2008). Do needs, symptoms or disability of outpatients with schizophrenia influence familiy burden? Social Psychiatry and Psychiatric Epidemiology, 43(8), 612-618.

Ohaeri, J. (2003). The burden of caregiving in families with a mental illness: a review of 2002. Current Opinion in Psychiatry, 16(4), 457-465.

Papastavrou, C., Charalambous, A., Tsangari, H. y Karayiannis, G. (2010). The cost of caring: the relative with schizophrenia. Scand J. Caring Sci., 24(4), 817-823.

Provencher, H. y Mueser, K. (1997). Positive and negative symptom behaviors and caregiver burden in the relatives with schizophrenia. Schizophrenia Research, 26(1), 71-80.

Rebolleda, C. y Soladana, L. (2008). Propuestas de abordaje en la intervención precoz de la Psicosis con usuarios de un CRPs y sus familias. Informaciones Psiquiátricas, $194,343-370$.
Reine, G., Lancon, C., Avorio, A., Duplan, S., Simeoni, M. C., Aghababian, V. y Auquier, P. (2004). Evaluation of burden in caregivers of patients suffering from schizophrenia. Dimensions and domains. Annales Medico Psychologiques, 162, 453-461.

Reine, G., Lancon, C., Simeoni, M. C., Duplan, S. y Auquier, P. (2003). Caregiver burden in relatives of persons with schizophrenia: an overview of measure instruments. Encephale, 29(2), 137-147.

Roick, C. et al. (2007). Burden on caregivers of people with schizophrenia: comparison between Germany and Britain. Br. J. Psychiatry, 190, 333-338.

Roick, C., Heider, D., Toumi, M. y Angermeyer, M. (2006). The impact of caregivers' characteristics, patients' conditions and regional differences on family burden in schizophrenia: a longuitudinal analysis. Acta Psychiatr Scand, 114(5), 363-374.

Rose, D., Thornicroft, G., Pinfold, V. y Kassam, A. (2007). 250 labels used to stigmatise people with mental illness. BMC Health Services Research, 7(97), 1-7.

Rummel-Kluge, C. y Kissling, W. (2008). Psychoeducation for patients with schizophrenia and their families. Expert Rev. Neurother, 8(7), 1067-1077.

Sales, E. (2003). Family burden and quality of life. Quality of Life Research, 12(Suppl 1), 33-41.

Schene, A. (1990). Objective and subjective dimensions of family burden. Towards an integrative framework for research. Soc Psychiatry Psychiatr Epidemiol, 25(6), 289297.

Schulze, B. y Rössler, W. (2005). Caregiver burden in mental illness: review of measurement, findings and interventions in 2004-2005. Curr Opin Psychiatry, 18(6), 684-691.

Tobío C. (2008). Redes familiares, género y política social en España y Francia. Política y Sociedad, 45(2), 87-104.

Tobío, C., Agulló, M., Gómez, M. y Martín, M. (2010). El cuidado de las personas. Un reto para el siglo XXI. Recuperado de http://obrasocial.lacaixa.es/deployedfiles/obrasocial/Estaticos/pdf/Estudios_sociales/ vol28_completo_es.pdf

Vidal, M., Cortés, M. J., Valero, J., Gutiérrez-Zotes, A. y Labad, A. (2008). Ambiente familiar y emoción expresada en pacientes con esquizofrenia $u$ otras psicosis y en sus familiares de primer grado. Actas Esp Psiquiatr, 36(5), 271-276. 